\title{
Tratamiento de estenosis biliares complejas
}

\author{
Rodrigo Soto-Solís* \\ Centro Médico Nacional 20 de Noviembre, ISSSTE, Ciudad de México, México
}

\begin{abstract}
Resumen
Sin duda los avances tecnológicos son uno de los grandes pilares de la medicina moderna. En esta segunda versión virtual de los Ecos Endoscópicos vemos un futuro esperanzador, en el que haremos reuniones que combinen la educación a distancia y la reunión presencial se están volviendo una realidad. Esta es una revisión analítica de las sesiones y los trabajos libres presentados en la Digestive Disease Week 2021 (DDW 2021) en donde se evaluó el tratamiento endoscópico de las estenosis biliares complejas.
\end{abstract}

Palabras clave: Radiofrecuencia biliar. Colangiopancreatografía retrógrada endoscópica (CPRE). Estenosis biliar hiliar. Prótesis biliares metálicas. Prótesis biliares plásticas.

\section{Introducción}

Las estenosis complejas de la vía biliar continúan siendo un reto en el que se ha logrado un gran avance en el tratamiento endoscópico-quirúrgico. Hay diferentes factores que intervienen en el éxito del tratamiento endoscópico.

\section{Objetivo}

Realizar una revisión analítica y crítica de los trabajos presentados en la Digestive Disease Week 2021 (DDW 2021), en donde se haya evaluado el tratamiento endoscópico de las estenosis biliares complejas. Trabajos incluidos: 18 . Evaluados: 28 carteles y 8 presentaciones orales.

\section{Para una MEJOR COMPRESIÓN, SE HA DIVIDIDO EN LOS SIGUIENTES SUBTEMAS}

1. Estenosis biliares por condición clínica específica: colangitis esclerosante primaria (CEP), trasplante hepático ortotópico (THO) y colangiocarcinoma (colangioCa).

2. Tratamiento endoscópico con tecnología en desarroIlo: Radiofrecuencia (RFA) y terapia fotodinámica (PDT).

3. Uso del ultrasonido endoscópico (EUS) en estenosis biliares complejas.

\section{Estenosis biliares por condición clínica específica}

\section{Colangitis esclerosante primaria (CEP)}

En el primer estudio que presentamos, se evaluó la dilatación con o sin la colocación de una prótesis plástica en la CEP. Se trata de un metaanálisis comparativo que conjunta la información de 10 estudios y 455 pacientes: 360 en dilatación con balón (BD) y 95 en dilatación con balón y prótesis (BDS) ${ }^{1}$. El éxito técnico para $B D$ fue de 96.8 y 91.9 para BDS con una $p<0.001$. El éxito clínico fue de 86.5 vs. $70.8 \%$ respectivamente $(p<0.001)$. Se trata del primer metaanálisis de este 
tipo sobre esta cuestión, nos da evidencia más fuerte para no dejar prótesis en este subgrupo de pacientes, aunque la heterogeneidad fue de moderada a sustancial. El segundo estudio es también un metaanálisis y revisión sistemática que fue presentado como póster y evalúa lo mismo. En este cartel incluyeron 4 estudios, 394 pacientes, 250 con prótesis y 144 solo dilatación. Solo un ensayo clínico y no hubo diferencias en cuanto a efectividad, pero sí mayor colangitis en el grupo con prótesis $(p<0.0001)^{2}$. En otro estudio de la Clínica Mayo de Rochester evaluaron el uso de balones de calibre pequeño para estenosis complejas (biliares y pancreáticas). Se trata del uso de balones de angioplastia (3 - $4 \mathrm{~mm}$ ), primordialmente en pacientes con CEP (25/45). El éxito técnico fue del $91.7 \%$ y los autores concluyen que se trata de una opción útil para este tipo de estenosis ${ }^{3}$.

\section{TRASPLANTE HEPÁtICO ORTOTÓPICO (THO)}

En estenosis biliar postrasplante en pacientes con CEP se comparó la terapéutica percutánea vs. la endoscópica. A priori me parece que no hay duda en que la primera línea de tratamiento debería ser la endoscópica, lo cual concuerda con lo que demuestran los autores de la Universidad de Colorado al evaluar una cohorte de 108 pacientes con THO por CEP, de los cuales 30 requirieron intervención biliar' ${ }^{4}$.

El quinto estudio que he incluido en este resumen evaluó la utilidad de las prótesis plásticas vs. las metálicas en la rehabilitación de las estenosis biliares postrasplante ${ }^{5}$. Se trata de un estudio retrospectivo realizado en el hospital presbiteriano de Nueva York que incluyó 48 pacientes y en el cual no lograron encontrar diferencia alguna entre el uso de prótesis metálicas vs. plásticas.

\section{Colangiocarcinoma (ColangioCa)}

La evidencia en favor de las prótesis metálicas en colangioCa es sólida. En el MD Anderson presentaron su experiencia en pacientes con colangioCa y prótesis metálicas y hacen un análisis comparativo con prótesis plásticas. Es un estudio retrospectivo con 74 pacientes, 19 con prótesis metálicas y 55 con plásticas. Se utilizaron principalmente prótesis descubiertas en pacientes con Bismuth I y II. Concluyen que disminuyen el número de intervenciones y tienen mayor duración; sin embargo, en colangioCa hiliares muchas veces no se utilizan prótesis metálicas por la supervivencia más larga ${ }^{6}$.

Los siguientes estudios incluidos son sobre las estenosis hiliares, las cuales desde mi perspectiva son las más difíciles de tratar. En una revisión sistemática y metaanálisis que incluyó 10 estudios y 1,529 pacientes para determinar si hay diferencias claras entre el drenaje unilateral y bilateral ${ }^{7}$. Los autores concluyen que el drenaje bilateral muestra un menor éxito técnico con mayor éxito clínico. Las reintervenciones y las complicaciones asociadas son similares. El siguiente estudio japonés evalúa una novedosa prótesis metálica descubierta cuya configuración de permite su apertura de 4 a $10 \mathrm{~mm}$ en cualquier sitio para poder pasar otra prótesis en el drenaje bilateral hiliar con técnica de prótesis dentro de la prótesis. Son 33 pacientes y muestra que es técnica mente factible y clínicamente útil ${ }^{8}$.

Aspectos técnicos que se evalúan en el drenaje de malignidad hiliar incluyen la técnica de prótesis dentro de prótesis (SIS) o prótesis a un lado de la prótesis (SBS). En el Hospital Universitario Thomas Jefferson de Filadelfia realizaron este estudio retrospectivo con 158 pacientes a los que se les colocaron prótesis metálicas descubiertas de forma bilateral y con técnica SBS con extensión a duodeno sin reintervención en 129 días $^{9}$. En la presentación oral se mostraron los datos de los 44 pacientes que requirieron reintervención: 44 sujetos 1 evento, 22 requirieron 2 eventos y 9 sujetos 3 eventos. Lo más interesante, en que se utilizó terapia térmica para recanalizar el $\operatorname{conducto}^{10}$. En el metaanálisis y revisión sistemática de la Universidad de Sao Paulo de Brasil, evaluaron diferencias entre la técnica SIS vs. SBS. Incluyeron 4 estudios con 186 pacientes y, aunque no encontraron diferencias claras, en el grupo de SIS hubo una mayor reducción de las cifras de bilirrubina sin impacto clínico alguno ${ }^{11}$.

\section{Tratamiento endoscópico con tecnología en desarrollo}

\section{RAdiofreCUENCIA}

Recientemente ha aumentado la información sobre la utilidad de la radiofrecuencia biliar, puesto que el $80 \%$ del colangiocarcinoma se diagnostica en etapas avanzadas 0 irresecables. En estos dos metaanálisis, investigadores de la Universidad de Illinois en Chicago nos presentan la utilidad paliativa y su impacto positivo en la supervivencia $\left(4\right.$ meses) ${ }^{12,13}$.

\section{TERAPIA FOTODINÁMICA}

La terapia fotodinámica se encuentra disponible en muy pocos centros. En algunos estudios ha mostrado ser útil como medida paliativa en el colangioCa irresecable. 
Los datos de 4 estudios y 255 pacientes en este metaanálisis compararon la quimioterapia sistémica vs. quimioterapia sistémica y terapia fotodinámica. Encontraron una mayor supervivencia global (3.34 meses) y al año ${ }^{14}$.

\section{USO DEL ULTRASONIDO ENDOSCÓPICO EN ESTENOSIS BILIARES COMPLEJAS}

Investigadores de Michigan evaluaron la duración del drenaje transpapilar vs. la coledocoduodenostomía ${ }^{15}$. Se trata de un estudio retrospectivo de 15 casos hechos con ultrasonido endoscópico y prótesis de aposición luminal contra 30 por vía transpapilar. El tiempo de utilidad del drenaje fue similar. El siguiente estudio evalúa la colecistogastro o colecistoduodenostomía guiada por ultrasonido endoscópico para la obstrucción biliar distal. El éxito clínico en 46 pacientes fue del $80 \%$ y el técnico del $100 \%{ }^{16}$. En otro estudio evaluaron la utilidad de la hepaticogastrostomía para las enfermedades biliares; destaca que 4 de los 15 evaluados tenían enfermedad benigna. El éxito técnico fue del $93 \%{ }^{17}$.

Finalmente, se presentó el algoritmo terapéutico en Osaka para las estenosis de las hepaticoyeyunoanastomosis con la ayuda del USE. Proponen la hepaticogastrostomía de acceso y tratamiento anterógrado. Evaluaron 33 pacientes y 14 de estos requirieron prótesis metálica por vía anterógrada ${ }^{18}$.

\section{Conclusiones}

En el último año se han presentado avances en el tratamiento de las estenosis biliares complejas. Desde mi punto de vista las dos tecnologías que están mejorando el drenaje son el ultrasonido endoscópico y la radiofrecuencia biliar.

\section{Financiamiento}

La Asociación Mexicana de Endoscopia Gastrointestinal financió mi participación en la Semana de Enfermedades Digestivas en modalidad virtual (DDW) para la realización de este trabajo.

\section{Conflicto de intereses}

Durante los últimos tres años, con o sin el pago de honorarios, declaro que he hecho consultorías, he dado pláticas y he realizado procedimientos, lo cual puede verse como un potencial conflicto de intereses: Ovesco Endoscopy AG, Boston Scientific y Ferring Pharmaceuticals México.

\section{Bibliografía}

1. Dhaliwal A, Saghir S, Daryl R, Naga Y, Dhindsa B, Ofosu A, et al. Endoscopic balloon dilation vs. endoscopic balloon dilation with stenting for dominant strictures in primary sclerosing cholangitis: A comparative meta-analysis. Presentación oral (390131), DDW 2021.

2. Beran A, Ghazaleh S, Al-abboodhi Y, Mhanna M, Ayesh H, Srour O, et al. Balloon dilation vs. stent placing for dominant strictures in primary sclerosing cholangitis: A systematic review and meta-analysis. DDW 2021.

3. Bailey NJ, Topazian, Martin JA, Storm AC, Abu Dayyeh BK, Levy MJ, et al. ID: 3525682 In a Pinch: Use of small caliber dilating balloons during ERCP for salvage treatment of high-grade biliary and pancreatic duct strictures. Gastrointest Endosc [Internet]. 2021;93(6 Suppl):AB137. Disponible en: https://www.giejournal.org/article/S0016-5107(21)01203-7/ abstract

4. Jonica ER, Han S, Shah RJ. ID: 3520602 Endoscopic vs. percutaneous management of post-transplant biliary strictures in primary sclerosing cholangitis patients. Gastrointest Endosc [Internet]. 2021;93(6 Suppl):AB127-AB128. Disponible en: https://www.giejournal.org/article/ S0016-5107(21)00548-4/fulltext

5. Hajifathalian K, Skaf DA, Katz PO, Samstein B, Brown RS, Fortune BE, et al. Treatment of biliary anastomotic stricture after liver transplantation: is metal better than plastic? ePoster, Fr417, DDW 2021.

6. Emelogu IK, Kumar S, Yu AC, Coronel M, Coronel E, Ge PS, et al. ID: 3523951 Metal biliary stents for biliary obstruction in cholangiocarcinoma. Gastrointest Endosc [Internet]. 2021;93(6 Suppl):AB126-AB127. Disponible en: https://www.giejournal.org/article/S0016-5107(21)00546-0/abstract

7. Ghazaleh S, Beran A, Abulhelwa Z, Aziz M, Elzanaty AM, Sharma S, et al. ID: 3520437 Bilateral vs. unilateral stenting for unresectable malignant hiliar biliary obstruction - A systematic review and meta-analysis. Gastrointest Endosc [Internet]. 2021;93(6 Suppl):AB123. Disponible en: https://www.giejournal.org/article/S0016-5107(21)00541-1/pdf

8. Ogura T, Yamada M, Ueno S, Yamada T, Okuda A, Nishioka N, et al Su1516 Single-session multiple stent deployment using a novel stent without dilating initial stent mesh to treat malignant hepatic hiliar obstruction. Gastrointest Endosc [Internet]. 2021;93(6 Suppl):AB360. Disponible en: https://www.giejournal.org/article/S0016-5107(20)32602-X/fulltext

9. Chalikonda D, Mitsuhashi S, Bashir M, Holmes I, Chiang A, Schlachterman A, et al. ID: 3526477 Bilateral biliary drainage for malignant hiliar obstruction using side-by-side uncovered metal stents: Patients demographics and predictors of clinical outcomes. Gastrointest Endosc [Internet]. 2021;93(6 Suppl):AB124. Disponible en: https://www.giejournal.org/ article/S0016-5107(21)00543-5/pdf

10. Biliary stenting for hiliar malignant biliary obstruction: Endoscopic management of bilateral uncovered metal stent occlusion and outcomes. Presentación oral, DDW 2021.

11. Souza G, Ribeiro I, Funari M, De Moura D, Scatimburgo MV, De Freitas Junior J, et al. ID: 3520569 Endoscopic bilateral drainage techniques of malignant hiliar biliary obstruction: Stent-in-stent or side-by-side? A systematic review and meta-analysis. Gastrointest Endosc [Internet]. 2021;93(6 Suppl):AB135. Disponible en: https://www.giejournal.org/article/S0016-5107(21)01200-1/pdf

12. Rebhun J, Villa E. Biliary ERFA in palliation of unresectable cholangiocarcinoma: A meta-analysis on performance outcomes and safety profile. ePoster, DDW 2021.

13. Rebhun J, Villa E. Biliary endoscopic radiofrequency ablation and percutaneous radiofrequency ablation lead to improved median and mean survival in patients with unresectable cholangiocarcinoma: A meta-analysis. ePoster, DDW 2021.

14. Nassani N, Ghoulam E, Siddiqui UD, Villa E. ID: 3523646 Photodynamic therapy alone vs. photodynamic therapy combined with systemic chemotherapy for unresectable extrahepatic cholangiocarcinoma: A pooled analysis. Gastrointest Endosc [Internet]. 2021;93(6 Suppl):AB128. Disponible en: https://www.giejournal.org/article/S0016-5107(21)01186-X/pdf

15. Nemakayala DR, Reif M, Shah JN, Evans J, Romero R, El Chafic AH. Biliary drainage patency following ERCP - placed transpapillary self - expandable metal stents vs. EUS-guided choledocoduodenostomy with lumen - apposing metal stents for malignant biliary obstruction. ePoster Su224, DDW 2021.

16. Binda C, Anderloni A, Fugazza A, Amato A, de Nucci G, Redaelli A, et al. ID: 3523449 EUS-gallbladder drainage using lumen-apposing metal stent as rescue treatment for malignant distal bilary obstruction: A large multicenter experience. Gastrointest Endosc [Internet]. 2021:93(6 Suppl):AB244-AB245. Disponible en: https://www.giejournal.org/article/ S0016-5107(21)00771-9/fulltext

17. Dorrell R, Pawa R. Safety and efficacy of endoscopic ultrasound-guided hepaticogastrostomy for management of biliary disease. ePoster, DDW 2021.

18. Yamada M, Ogura T, Komori T, Nishioka N, Okuda A, Ueno S, et al. Sa1418 Innovative treatment technique for hepaticojejunotomy stricture using novel fine-gauge electrocautery dilator. Gastrointest Endosc [Internet]. 2021;91(6 Suppl):AB180-AB181. Disponible en: https://www.giejournal.org/article/S0016-5107(20)31391-2/fulltext 